\title{
Study on the Cultivation of Clinical Ability of Postgraduates Majoring in Clinical Medicine
}

\author{
Zhong Zhihong ${ }^{1}$, Yan $\mathrm{Jun}^{2}$, Li Xia ${ }^{1, *}$ \\ ${ }^{1}$ Gannan Medical University, 341000 \\ ${ }^{2}$ First Affiliated Hospital of Gannan Medical University, 341000 \\ *Corresponding author
}

Keywords: clinical medicine; degree; postgraduate; clinical ability

\begin{abstract}
Taking the postgraduates of master degree in clinical medicine of local medical colleges as the research object, the common problems in the cultivation of clinical competence for postgraduates of clinical medicine master degree under the combination of postgraduate training of clinical medicine and standardized training of residents, and suggested improvements are described in this paper.

Postgraduate education in master degree in clinical medicine is an important part of higher medical education in China. Clinical competence training, as the core of postgraduate training for master degree in clinical medicine, is the key factor in determining the quality of postgraduate training in clinical medicine master degree. The cultivation of the clinical abilities and practical guidance are provided in this paper.
\end{abstract}

\section{Objects and Methods}

Gannan Medical University graduated in 2015 and 2016 with a master's degree in clinical medicine.

Self-made "Clinical Ability Training of Postgraduates in Clinical Medicine Master Degree Survey Form”,30 graduate students of master degree in clinical medicine were selected to conduct a questionnaire survey.

The survey includes: basic conditions, knowledge of the current clinical competence training, communication with patients during the clinical rotation process, examination of licensed physicians, standardized training of resident physicians, and publication of papers.

\section{Results}

A total of 30 questionnaires were issued and 30 were recovered. The recovery rate was $100 \%$. Questionnaires with a vacancy rate of more than 50\%, or with apparently logical errors, are considered invalid and all questionnaires are valid, with an effective rate of $100 \%$.

In 2015, the school enrolled 11 postgraduates of clinical medicine master degree, 19 were recruited in 2016, and 30 people were enrolled for two years, including 20 boys and 10 girls, enrolled in professional programs covering internal medicine, surgery, pediatrics, imaging medicine and nuclear medicine, anesthesiology, dermatology and venereal disease, oncology, and nursing.

According to the survey, $73.33 \%$ of postgraduate students stated that they were "more explicit" or "very clear" about the goal of clinical medical degree training. $76.67 \%$ of graduate students believe that the purpose of clinical competence training is to improve clinical skills. The top three positions in the form of postgraduate clinical ability training are: case teaching and surgery, group discussion, and expert report progress. $80 \%$ of postgraduate students pay more attention to or pay more attention to their own clinical ability. $73.33 \%$ of postgraduate students believe that their clinical abilities are "more skilled" or "very skilled". In the rotation practice, $80 \%$ are temporary arrangements to teach. $76.67 \%$ of the rotating departments "sometimes have" or "always have" 
arrangements to organize experts to use lectures to improve clinical theory. All graduate students indicated that they can complete clinical practice work in strict accordance with the training plan. During the clinical rotation, $40 \%$ of graduate students said that "most of them can" or "can" manage beds individually. 53.33\% of postgraduates manage beds 3-5. During the clinical rotation, 76.67\% of postgraduate students said that most of them were capable of performing diagnostic procedures and $15.79 \%$ of postgraduate students could participate in daily operations. $76.67 \%$ of graduate students can participate in the rescue of critically ill patients. $86.67 \%$ of graduate students have the opportunity to complete the four punctures during clinical rotation. $76.67 \%$ of postgraduate students were able to complete CPR skills more skillfully or very skillfully during clinical rotation. $63.33 \%$ of graduate students said that the rotating department conducts examinations. $56.67 \%$ of graduate students indicated that their examinations were mainly conducted by teachers, and $63.33 \%$ of postgraduate students showed that the examinations were the same as the standardized training for residents. $86.67 \%$ of postgraduate students think that clinical rotation record and fill in the clinical ability training rotation assessment form are reasonable. $93.33 \%$ of graduate students said they participated in the consultation work in the department. $66.67 \%$ of graduate students expressed their participation in the design work of scientific research projects in their units. $56.67 \%$ of graduate students said they participated in academic activities at least once a month. $76.67 \%$ of postgraduate students believe that after training, clinical ability "has a certain" or "significantly" increase. 46.67\% of graduate students believe that the management of current clinical arguments is "better" or "good". $53.33 \%$ of postgraduate students are "more satisfied" or "satisfied" with the overall professional graduate clinical ability training model. $80 \%$ of postgraduate students believe that the cultivation of clinical medicine professional postgraduates and the integration of standardization training in hospitalization are " some help" or "helpful" in cultivating the professional degree clinical competence.

83.33\% of graduate students believe that the current doctor-patient relationship is "very bad" or "very general." All graduate students believe that good communication can reduce the incidence of medical disputes. 93.33\% of postgraduates said they had received a course on "Doctor-patient communication" or lectures before clinical practice. $80 \%$ of graduate students indicated that they had received a course on "medical-patient communication" or lectures during clinical practice. $40 \%$ of postgraduate students are timid, nervous, and anxious when communicating with patients. 30\% of graduate students have insufficient confidence in their interaction with patients. 93.33\% of postgraduate students in the communication with patients, pay attention to the language to be easy to understand. The main factors that affect communication with patients are the lack of clinical communication experience and the lack of patient trust in students. The top three effective ways to improve the communication between doctors and patients are: teaching teachers to infiltrate the clinical teaching process, their own clinical practice, and experience exchanges.

(1) Publication of thesis of postgraduates of master degree in clinical medicine

The dissertation is a summary and summary of the comprehensive quality cultivation process for postgraduates of clinical medicine master degree, and it is an important part in the cultivation of graduate students. Of the 30 students in grades 2015 and 2016, 13 published 21 papers as the first author, including 6 core journals, 1 Chinese journal, and 14 other journals. The content of the article is related to review, clinical research type research, meta analysis, case studies and so on.

(2) Approved qualifications of certified professional medical graduates in clinical medicine

The postgraduate of a master's degree in clinical medicine at the school must take the National Practitioner qualification exam one year after enrollment as an annual assessment at the end of the first year of training. After the graduate student obtains the "certificate of practicing physician", he may engage in clinical work as a clinician and conduct systematic and standardized training of clinical ability, which in turn can improve the quality of clinical ability of clinical medical postgraduates. $96.67 \%$ of postgraduates passed the practicing physician exam, and $73.33 \%$ of postgraduates passed the resident physician standardized training assessment. 


\section{Discussion and Suggestions}

In 2015, the school enrolled 11 postgraduates of clinical medicine master degree. In 2016, it enrolled 19 people. The number of enrollment has nearly doubled. The school is continuously deepening the reform of postgraduate education and teaching, and actively promotes postgraduate training in clinical medicine master degree and standardized training for residents. Organic convergence. This is in line with the content requirements of the "Notice on Strengthening the Coordinating the Education and Teaching of Postgraduates of Clinical Master Degree with the Standardized Training of Resident Doctors" issued by the General Office of the Ministry of Education in $2016^{[1]}$.

The survey shows that $80 \%$ of graduate students pay more attention to or pay more attention to their own clinical ability. It shows that the postgraduates of the master degree in clinical medicine have not paid enough attention to the training of clinical abilities, and some students have neglected the essence of clinical medicine professional degree education as an occupational degree.The top three positions in the form of postgraduate clinical competence training are: case teaching and surgery, group discussion, and progress of expert reports. Case teaching is based on the content of teaching materials, selecting a representative medical record, guiding students to analyze the cases according to their knowledge, combining theory with practice, analyzing and summarizing cases, simulating the thinking process of treating patients in clinical work, and cultivating students. The clinical thinking ability lays the foundation for future clinical work ${ }^{[2]}$. The group discussion is to select a disease category before each discussion. Before the discussion, the team members should consult relevant books or literature on the etiology, pathology, pathogenesis, clinical manifestations, diagnosis and treatment of the disease, and conduct a retrospective review. Learn and select a student as the speaker. During the discussion, the lecturer will explain the learning points of the disease, and other students will ask questions or supplement. After discussion, the team members came up with the results of the discussions. Finally, the teachers were taught to comment on the group discussion. Through the group discussion, the graduate students deepened their impressions and understanding of the disease and accumulated more clinical experience.

93.33\% of graduate students said they participated in the consultation work in the department. Kee Nei's consultation is a case of critical illness or other cases with scientific research and teaching value. It is proposed by the attending physician, and the chief physician or department chief is involved in the consultation and discussion to further clarify and discuss the treatment advice. At the time of consultation, medical records are reported by the treating physician and the situation is analyzed, and the records of the consultation are fully completed. During this period, graduate students can learn how to accurately and completely report medical records, consider the causes, pathologies, diagnosis, and treatment of difficult and critical cases ${ }^{[3]}$.

$56.67 \%$ of graduate students said they participated in academic activities at least once a month. Academic lectures cover a wide range of topics, including diagnosis and treatment of diseases, clinical applications of advanced equipment and equipment, and scientific research project declarations. Through regular participation in academic activities, postgraduates understand current research in the field of clinical medicine and explore the horizon ${ }^{[4]}$.

63.33\% of postgraduate students said that the rotating departments perform examinations. The existing department assessments are mostly based on the normal performance of graduate students to score their clinical abilities. There are no quantification of assessment indicators and standards, and in particular there is no specific work on the clinical competence of graduate students. The evaluation of the indicators only lists assessment contents such as work attitude and work ability. There is no evaluation criterion, and the subjective factors of the examiners have a great influence. The mastery of basic knowledge, basic theories and basic skills during postgraduate clinical rotation lacks quantitative and objective indicators for assessment. In addition, the school management department failed to supervise the assessment of graduate students. There may be the possibility of distortion of the assessment process or assessment results, resulting in the assessment of some rotation departments in the form of going through the motions, and it is difficult to guarantee the 
quality of postgraduate clinical rotation ${ }^{[5]}$. Therefore, it is suggested that relevant management departments of the school should issue assessment criteria, formulate quantitative assessment indicators, and the clinical department strictly implement the assessment system of the department, and make a true assessment of the quality and effectiveness of clinical demonstration training for graduate students. By strengthening the evaluation of postgraduate clinical ability training, the purpose of taking examinations and promoting learning is achieved, and the progress is gradually progressed, thereby promoting the continuous improvement of its clinical ability ${ }^{[6]}$.

Among the 21 papers published by the postgraduates of the master degree in clinical medicine as the first author, there are 15 articles reviewed, 3 clinical research articles, 1 meta analysis, and 2 case studies. The article is mainly based on review, that is, a certain period of time, the author categorizes and analyses the data, data, and main points of a large number of original research papers for a certain topic, and analyzes the papers written and refined. As a clinical postgraduate with a master degree in clinical medicine, there is a lack of clinically applicable papers, and only theoretical research or research papers are not sufficient for their training requirements. Under the guidance of a tutor, it is necessary to complete the clinical work by combining the actual work of their own clinical departments. The practical problems encountered are the research subjects' papers, summarizing and summarizing the new technologies and methods to solve this problem, and promoting the systematic and standardized cultivation of the clinical competence for postgraduates of clinical medicine master degree programs, and realizing the transfer from medical students to doctors. change.

93.33\% of postgraduates said they had received a course on "medical-patient communication" or lectures before clinical practice. $80 \%$ of postgraduate students said they received relevant learning during clinical practice. Tip When graduate students enter the clinical practice, they should pay attention to and increase the training of their doctor-patient communication skills. Regularly invite experts in doctor-patient communication to the lecture or lecture, and organize the exchange of doctor-patient communication experience to make the graduate students understand some of the patients. Communicating actual combat experience, or conducting discussion-based teaching on a typical clinical case to improve postgraduate doctor-patient communication skills.

$40 \%$ of postgraduate students are timid, nervous, and anxious when communicating with patients. $30 \%$ of graduate students have insufficient confidence in their interaction with patients. In response to this phenomenon, schools can use role-playing to cultivate postgraduate doctor-patient communication skills, allow students to think about transpositions, enhance their understanding of the role of others ${ }^{[7]}$, and improve their relationship building, information collection, and verbal communication. The ability ${ }^{[8]}$.

\section{Acknowledgement}

Fund Project: 2016 Jiangxi Province Degree and Graduate Education Teaching Reform Research Project (Project ID: Jxyjg-2016-156).

\section{References}

[1] Li Jihong, Liu Fuquan. The current situation and countermeasures for the cultivation of master's degree in clinical medicine under "medical education cooperation" [J]. Chinese Journal of Medical Education Exploration, 2017, 16(11):1081-1085.

[2] Li Chunyan, Wang Yingyan, Lu Shuming, et al. Case teaching in clinical medicine professional degree graduate students in the application of clinical teaching [J]. Medical Theory and Practice, 2014, 27(24): 3370-3372.

[3] Cai Xiuying, Ni Jianqiang, Duan Xiaoyu, et al. Cultivation of practical ability for postgraduates in clinical medicine [J]. Journal of Nanjing Medical University (Social Science Edition), 2013, 57 (4):367-369.

[4] Shi Chujun, Xu Jiezhou, Yang Jinhua, et al. To construct a multi-teaching system for clinical 
skills of medical students and explore new models for the cultivation of innovative medical personnel [J]. Modern Medicine and Health, 2012, 28(19): 3010-3011.

[5] Hu Weili, Chen Yiting, Xie Peng, et al. Based on the "5+3" reform to strengthen the clinical medicine master degree graduates clinical ability training difficulties and countermeasures [J]. Degree and Graduate Education, 2016, 8:29-33.

[6] Li Minge. The practical research of combination of postgraduate education in clinical medicine master degree and standardized training of resident physicians [J]. Chinese Journal of Medical Education, 2017, 37(2): 286-290.

[7] Calhoun A,Rider E, Meyer E,et a1.Assessment of communication skills and self-appraisal in the simulated environment: feasibility of multirateral feedback with gapanalysis[J].Simul Healthe, 2009, $4(1): 22-29$.

[8] Li Yong, Wang Jinfan, Xu Nianzhen, et al. The role of role-playing method in the training of medical students' ability of doctor-patient communication [J]. Chinese General Practice Medicine, 201l, 9(11):1809-1810. 